\title{
Erectile dysfunction among male patients diagnosed with schizophrenia being treated with antipsychotic medication, and the impact on quality of life
}

\author{
WWJSM Rowel, ULNS Liyange, UH Hewawitharana, M Dayabandara, A Rodrigo
}

\section{Introduction}

Erectile dysfunction is a distressing side effect of antipsychotics, which leads to poor medication compliance and poor quality of life. There is a scarcity of studies on antipsychotic-induced sexual dysfunction in Sri Lanka. We assessed the prevalence of erectile dysfunction among patients with schizophrenia who have been prescribed antipsychotic medication.

\section{Methods}

A cross sectional, descriptive study was conducted among patients attending the outpatient clinics of Colombo North Teaching Hospital and National Hospital, Sri Lanka. The five item International Index for Erectile Dysfunction, Erection Hardness Score and World Health Organization Quality of Life Brief Scale were used to assess erectile dysfunction and quality of life.

\section{Results}

Of the 102 patients in the study, 80 (79\%) were found to have erectile dysfunction. The largest proportion of erectile dysfunction was seen in patients who were prescribed clozapine (87.9\%), followed by risperidone (82.1\%), olanzapine (73.9\%) and fluphenazine $(68.8 \%)$. There was no significant correlation between erectile dysfunction and quality of life. None of the patients had initiated a discussion about sexual side effects, while only $8.3 \%$ reported that their psychiatrist had inquired about the sexual dysfunction.

\section{Conclusions}

The results of this study suggest that sexual dysfunction is a common but often overlooked phenomenon among patients with schizophrenia. This study highlights the need for Sri Lankan psychiatrists to explore sexual problems in this cohort of patients.

SL J Psychiatry 2018; 9(2): 10-13

\section{Introduction}

Sexual dysfunction is a common and serious public health concern. A community study conducted in the United States reported that more than $40 \%$ of women and nearly $30 \%$ of men had some form of sexual dysfunction, with low sexual desire in women (22\%) and premature ejaculation in men (21\%) being the most prevalent (1). The Massachusetts male ageing study reported a 52\% prevalence of erectile dysfunction among males aged between 40 to 70 years (2). A Sri Lankan study reported a $73.1 \%$ prevalence of erectile dysfunction among people with diabetes mellitus (3).

Sexual dysfunction is often experienced by people with psychiatric illness and commonly occurs in patients with schizophrenia. An international study conducted across 27 countries revealed that the prevalence of sexual dysfunction among patients with schizophrenia was approximately 50\% [4]. Sexual dysfunction in patients with schizophrenia may be related to the symptoms of the disease, such as negative symptoms, psychosocial factors such as social isolation, physical health problems such as diabetes and the use of psychotropic medication. Among these factors, sexual dysfunctions secondary to antipsychotics is considered to be a leading cause.

Sexual dysfunction is considered to be one of the most common and distressing side effects of antipsychotic medication (5-7). Both first and second generation antipsychotic medications are associated with sexual dysfunction. Generally, first generation antipsychotic medications have a higher tendency to induce sexual dysfunction, with nearly half (45\%) of patients prescribed first generation antipsychotics experiencing some degree of sexual dysfunction (8). However, a high prevalence of 
sexual dysfunction has also been reported with risperidone (43.2\%) followed by haloperidol (38.1\%), olanzapine (35.3\%) and quetiapine (18.2\%) (9). Antipsychotic related sexual side effects include impaired libido, orgasmic dysfunction, and erectile and ejaculatory dysfunction (5). Erectile dysfunction is one of most common sexual dysfunction in men who are prescribed antipsychotics, with 38\% reporting difficulties in achieving erection, while $42 \%$ had difficulty maintaining erection (10). Consequently, it is likely that sexual side effects have a profound impact on quality of life and compliance to prescribed antipsychotic medications (11).

While this area has been thoroughly investigated in the West, there is a dearth of such studies in non-western settings including in Sri Lanka. It is of key importance to study this phenomenon in countries such as Sri Lanka, particularly because patients are unlikely to report sexual side effects on their own, unless inquired, due to issues such as embarrassment and stigma, and clinicians in turn may often overlook possible sexual side effects. Improved awareness of antipsychotic related sexual side effects in the Sri Lankan setting may lead to better recognition and management of same, which in turn may have a positive impact on the course of illness by improving both compliance and quality of life.

\section{Methods}

We conducted a cross sectional descriptive study to explore the prevalence of erectile dysfunction among male patients with schizophrenia, who were in remission at the time of the study, and who were taking antipsychotic medication.

Male patients diagnosed to have schizophrenia after assessment by a consultant psychiatrist according to ICD 10 criteria, who had been on antipsychotic medication for 3 months or more, were recruited to the study. Patients with acute psychotic or mood symptoms or current substance misuse were excluded from the study. Patients with a diagnosis of sexual dysfunction prior to commencement of antipsychotic treatment, those who were on more than one antipsychotic medication, and those on other medications affecting sexual function, such as benzodiazepines, antidepressants or antihypertensives were also excluded. All eligible patients who attended the psychiatry outpatient clinics of the National Hospital of Sri Lanka and Colombo North Teaching Hospital, Ragama, on conveniently selected days in the months of July, October and November 2018 were invited for the study.

Confirmation of the diagnosis of schizophrenia and details of currently prescribed medication were obtained by perusal of case records. A brief clinical interview was conducted with participants to exclude acute symptoms and substance misuse.
Information was gathered using a self-administered questionnaire to ascertain demographic and clinic details, as well as three self-report instruments. The five item International Index for Erectile Dysfunction (IIEF-5) and the single item Erection Hardness Score (EHS) were used to assess erectile dysfunction while the World Health Organization Quality of Life Brief Scale (WHOQOLBREF) was used to assess quality of life. The IIEF and EHS are scales with good psychometric properties and a high sensitivity and specificity for detecting treatmentrelated changes in patients with erectile dysfunction $(12,13)$. The WHOQOL-BREF explores many subjective aspects of quality of life, and is validated for use in patients with schizophrenia (14).

We inquired from selected patients whether they had discussed the sexual side effects they had experienced, with the psychiatrist or treating doctor, and whether the psychiatrist or treating doctor had inquired about the sexual side effects at any time during clinical reviews over the last 12 months.

The questionnaires were available in English, Sinhalese and Tamil languages and patients were given the choice of completing the questionnaire in their preferred language. When required, patients were given assistance to complete the questionnaires.

Considering the sensitive nature of the questions, adequate privacy was ensured when answering the questions. Informed written consent was obtained from the participants prior to inclusion in the study. Ethical clearance for the study was obtained from the Ethics Review Committee of the National Hospital of Sri Lanka.

\section{Results}

One hundred and two patients took part in the study and the mean age of the population was 39.4 (SD 13.5) years. The mean duration of illness in the population was 9.1 (SD 7.8) years and the mean duration of treatment was 7.3 years (SD 6.2). The mean duration of use of current medication was 1.2 years. Most patients were on clozapine ( $n=33,32 \%)$, followed by risperidone $(n=28$, $27 \%$ ), olanzapine ( $n=23 ; 23 \%)$, fluphenazine decanoate $(n=16 ; 16 \%)$ and aripiprazole $(n=2 ; 2 \%)$.

The mean score of the five item International Index for Erectile Dysfunction (IIEF-5) was 7.6 (SD 7.1) and mean Erection Hardness Scale (EHS) score 2.87 (SD 1.5). Accordingly, the prevalence of erectile dysfunction was $78.4 \%$.

The highest proportion of erectile dysfunction was seen in patients who were on clozapine (87.9\%), followed by risperidone (82.1\%), olanzapine (73.9\%) and fluphenazine $(68.8 \%)$. None of the patients on aripiprazole reported erectile dysfunction. 
The mean WHOQOL-BREF score for the whole population was 58.9 (SD 11.1). There was no significant correlation between the IIEF and WHOQOL BREF scores $(\mathrm{r}=0.41 \mathrm{p}=0.1)$. There was no significant correlation between the EHS (Erection hardness score) and WHOQOL scores $(r=0.35 ; \mathrm{p}=0.1)$. There was no significant difference between the average scores of the WHOQOL Brief among individuals with (56.7; SD 10.8) and without (60.1; SD 12.4) sexual dysfunction ( $p=0.2)$.

Only 24 participants of this study were asked about whether a discussion had ensued about sexual side effects during clinical reviews. None of the patients had initiated a discussion about their sexual side effects with the clinician, while only two patients (8.3\%) reported that their psychiatrist had inquired about possible sexual side effects.

\section{Discussion}

The results of this study indicate that erectile dysfunction is common among male patients with schizophrenia in Sri Lanka, yet surprisingly it did not appear to be associated with poor quality of life. Our study population had a higher prevalence of erectile dysfunction when compared to other studies $(10,15,16)$. This may at least partly be explained by the relatively longer duration of illness and older age of this study population. There is no available data to compare the findings of this study with rates of sexual dysfunction among the normative population in Sri Lanka. However, the prevalence of sexual problems in our study population was higher than that reported for diabetic patients in Sri Lanka (3).

It has been reported that clozapine produces a low incidence of hyperprolactinemia-related sexual dysfunction (15). Clozapine related sexual dysfunction is reported to be significantly lower when compared to patients treated with haloperidol and risperidone (16). However, in our study clozapine was associated with the highest prevalence of erectile dysfunction. This may be due to illness related factors rather than the antipsychotic medication. An Indian study has reported similar antipsychotic specific sexual dysfunction rates to this study, with a reported sexual dysfunction prevalence of $96 \%$ for risperidone, $90 \%$ for olanzapine and $88 \%$ for quetiapine (18).

Many patients with schizophrenia show an interest in sex that differs little from the general population (15). Yet, psychiatric symptoms, social isolation, and psychotropic medication often lead to sexual dysfunction. Thus it appears likely that sexual dysfunction in schizophrenia may have a close association with quality of life. However, we did not find a significant association between quality of life and sexual dysfunction in our study population. Our sample consisted of people with long history of schizophrenia who had been on antipsychotic medication for many years. The lack of sexual interest seen in this subgroup of individuals may explain the absence of a significant association between quality of life and sexual disfunction.

Sexual side effects are of major importance to patients and often lead to noncompliance to medication (11). Nonetheless, often neither patients nor doctors are willing to discuss sexual dysfunction (16). The sensitive nature of this topic may contribute to the reluctance to discuss this topic. Yet, when asked, most patients are not only willing to discuss about these problems, but feel relieved (16). It has been shown that discussions about sexual problems are not in any way counter therapeutic in patients with schizophrenia (16). In Sri Lanka, due to the nature of the doctor-patient relationship, patients are unlikely to discuss about it without being asked. None of the patients in this study had initiated a discussion about sexual side effects with their doctor, although most of them had experienced sexual side effects. Unfortunately, doctors often undervalue the importance of sexual problems and may not ask about this issue. The limited privacy and lack of adequate time for each patient in the Sri Lankan setting may also have been further reasons why only a very few doctors inquire about these problems.

\section{Limitations}

The validity of this study is limited by the small sample size, convenience sampling method and the study being conducted only in two clinics. The cross sectional nature of the study does not allow establishment of causality of the erectile dysfunction. We did not study the temporal relationship between sexual dysfunction and antipsychotic medication use, making it difficult to definitively conclude whether the erectile dysfunction is antipsychotic induced or not. We did not explore about medication compliance, which limits any observation between sexual dysfunction and medication adherence.

\section{Conclusions}

The results of this study suggest that sexual dysfunction is a common but often overlooked aspect of patients with schizophrenia. This study highlights the need for Sri Lankan psychiatrists to explore for sexual problems among this cohort of patients.

\section{Conflicts of interest}

None declared 
WWJSM Rowel, ULNS Liyange, UH Hewawitharana, University Psychiatry Unit, National Hospital, Colombo

M Dayabandara, Faculty of Medicine, University of Colombo, Colombo

A Rodrigo, Faculty of Medicine, University of Kelaniya, Ragama

Corresponding author: WWJSM Rowel

Email: madushi1985@yahoo.com

http://orcid.org/0000-0002-8819-430X

\section{References}

1. Laumann EO, Paik A, Rosen RC. Sexual dysfunction in the United States: prevalence and predictors. JAMA 1999; 281(6): 537-44.

2. Feldman HA, Goldstein I, Hatzichristou DG, Krane RJ, McKinlay JB. Impotence and its medical and psychosocial correlates: results of the Massachusetts Male Aging Study. J Urol 1994; 151(1): 54-61.

3. Malavige LS, Jayaratne SD, Kathriarachchi ST, Sivayogan S, Fernando DJ, Levy JC. Erectile dysfunction among men with diabetes is strongly associated with premature ejaculation and reduced libido. J Sex Med 2008; 5(9): 2125-34.

4. Dossenbach M, Hodge A, Anders M, et al. Prevalence of sexual dysfunction in patients with schizophrenia: international variation and underestimation. Int $\mathrm{J}$ Neuropsychopharmacol 2005; 8(2): 195-201.

5. Olfson M, Uttaro T, Carson WH, Tafesse E. Male sexual dysfunction and quality of life in schizophrenia. J Clin Psychiatry 2005; 66(3): 331-8.

6. Lambert M, Conus P, Eide P, et al. Impact of present and past antipsychotic side effects on attitude toward typical antipsychotic treatment and adherence. Eur Psychiatry 2004; 19: 415-22.

7. Montejo AL, Majadas S, Rico-Villademoros F, et al. Frequency of sexual dysfunction in patients with a psychotic disorder receiving antipsychotics. J Sex Med 2010; 7(10): 3404-13.
8. Smith SM O’Keane V, Murray R. Sexual dysfunction in patients taking conventional antipsychotic medication. $\mathrm{Br}$ J Psychiatry 2002; 181: 49-55.

9. Bobes J, Garc A-Portilla MP, Rejas J, et al. Frequency of sexual dysfunction and other reproductive side-effects in patients with schizophrenia treated with risperidone, olanzepine, quetiapine, or haloperidol: The results of the EIRE study. J Sex Marital Ther 2003; 29: 125-47.

10. Smith SM et al. Sexual dysfunction in patients taking conventional antipsychotic medication. Br J Psychiatry 2002.

11. Knegtering $H$, van den Bosch $R$, Castelein S, Bruggeman $\mathrm{R}$, Sytema S, van Os J. Are sexual side effects of prolactinraising antipsychotics reducible to serum prolactin? Psychoneuroendocrinology. 2008; 33(6): 711-7.

12. Rosen RC, Riley A, Wagner G, Osterloh IH, Kirkpatrick $\mathrm{J}$, Mishra A. The international index of erectile function (IIEF): a multidimensional scale for assessment of erectile dysfunction. Urology. 1997; 49(6): 822-30.

13. Mulhall JP, Goldstein I, Bushmakin AG, Cappelleri JC, Hvidsten K. Validation of the erection hardness score. J Sex Med. 2007; 4(6): 1626-34. Epub 2007 Sep 21.

14. Mas-Expósito L, Amador-Campos JA, Gómez-Benito J, Lalucat-Jo L; Research Group on Severe Mental Disorder The World Health Organization Quality of Life Scale Brief Version: a validation study in patients with schizophrenia. Qual Life Res. 2011; 20(7): 1079-89.

15. Serretti A \& Chiesa A. A meta-analysis of sexual dysfunction in psychiatric patients taking antipsychotics. International Clinical Psychopharmacology 2010; 26 : $130-40$.

16. de Boer MK, Castelein S, Wiersma D, Schoevers RA, Knegtering $\mathrm{H}$. The facts about sexual (Dys)function in schizophrenia: an overview of clinically relevant findings. Schizophr Bull 2015; 41(3): 674-86.

17. Atmaca M, Kuloglu M, Tezcan E. A new atypical antipsychotic: Quetiapine-induced sexual dysfunctions. Int J Impot Res 2005.

18. Nagaraj AK, Pai NB, Rao S. A comparative study of sexual dysfunction involving risperidone, quetiapine, and olanzapine. Indian J Psychiatry 2009; 51(4): 265-71. 